\title{
DWI before and after Gadolinium - EOB - DTPA administration: modifications of ADC maps evaluated with texture analysis.
}

\author{
Negrelli R. ${ }^{1}$, Cavalli D. ${ }^{1}$, Zamboni G.A. ${ }^{1}$, Boninsegna E. ${ }^{1}$, Cybulski A. ${ }^{1}$, Tambalo S. $^{2}$, Pozzi Mucelli R. ${ }^{1}$ \\ ${ }^{1}$ Department of Radiology, University Hospital of Verona; ${ }^{2}$ Center of Neuroscience and Cognitive Systems, University of Trento
}

\section{Purpose}

Diffusion weighted imaging (DWI) is now part of a routine abdominal MRI examination. It is commonly believed that the qualitative appearance of the DWI images and corresponding apparent diffusion coefficient (ADC) values of splanchnic organs are identical before and after the administration of gadolinium chelates contrast media.

- DWI is usually performed prior to the intravenous administration of a gadolinium chelates contrast agent.

In some situations it would be useful to perform DWI sequences after contrast medium injection: for the evaluation of focal liver lesions using Gadolinium-EOB-DTPA, multiple delayed phases are required, therefore it is desirable to administer contrast medium in the early part of the examination and after that, acquire delayed contrastographic phases.

- Gadolinium chelates may alter the T1 and T2 relaxation times, affecting values of ADC maps. A recent study conducted by Wang et Al. reported that gadolinium chelates do not alter both the ADC values and the qualitative appearance of abdominal viscera except for the kidneys. However it was conducted with Gd-BOPTA, whose excretion is mainly renal.

For the above-mentioned reasons we performed a specific evaluation of the modifications induced by Gd-EOB-DTPA in the DWI aspects and ADC values of the visceral organs. In addiction, we performed a texture analysis on the ADC maps, to depict alterations in mean values, Skeweness and Kurtosis induced by gadolinium chelates.

\section{Materials and Methods}

We reviewed the MRI examinations of 10 patients who underwent DWI before and after Gd-EOB-DTPA injection. We excluded patients who presented focal or diffuse lesions of the liver or other splanchnic organs alterations.

MR imaging was performed by means of a 1.5-T MR unit (Philips Ingenia; Eindhoven, The Netherlands) by using a phased-array coil. Patients were asked to fast for 6 hours before the MR. DWI was obtained using respiratory-triggered single-shot echo planar images, acquired at different $b$ values $(0-400-800 \mathrm{~s} /$ $\mathrm{mm}^{2}$ ); diffusion study was acquired before and 20 minutes after the injection of Gd-EOB-DTPA.

Qualitative image analysis was performed at a workstation by two radiologists (R.N., E.B., 6 and 4 years experience in gastrointestinal radiology, respectively). They assessed modifications of signal intensity of DWI images and corresponding ADC maps in the basal and in the late hepatobiliary phase. A MRI technologist (D.C., 10 years experience in MR abdominal imaging) positioned 5 ROIs within the liver parenchyma, 3 in the pancreatic parenchyma, 2 in the spleen, 1 in the non-dependent portion of the gallbladder, 2 in the renal cortex and 2 in the medulla of each kidney, 2 in the paraspinal muscles and 2 in the subcutaneous fat, to assess quantitative modifications in ADC values before and after administration of Gd-EOB-DTPA.

The mean ADC values were calculated by using ImageJ Software for Mac (version 10.2; National Institute of Health, USA).

Finally, texture analysis (mean, variance, skewness and Kurtosis) of these organs was performed on ADC maps before and after contrast medium injection, by using MaZda Software for Windows.

A paired t-test was applied to evaluate quantitative image analysis of the two groups, by using Prism Software for Windows.

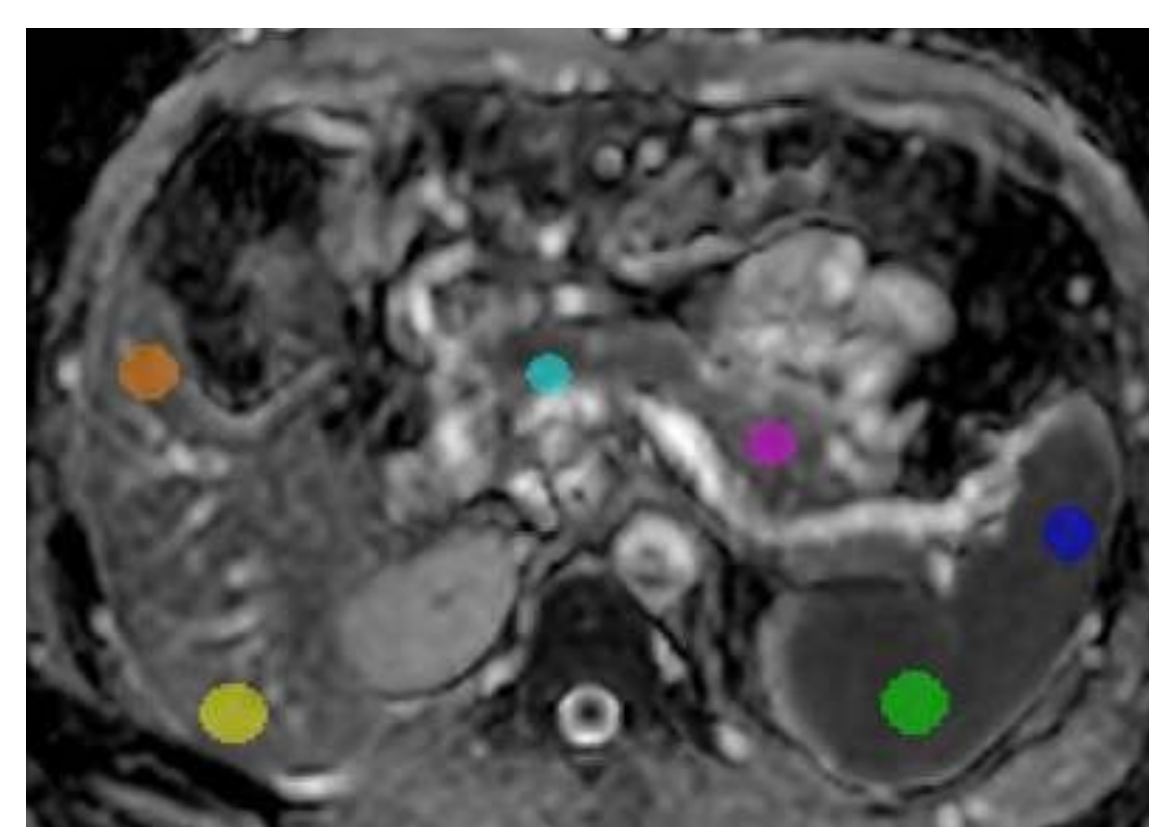

Results

In the qualitative image analysis, the radiologists did not depict any signal modifications in diffusion-weighted images and ADC maps of any splanchnic organ after Gd-EOB-DTPA administration.

The quantitative values of ADC mean values before and after Gd-EOB-DTPA injection are summarized in Fig. 1.

\begin{tabular}{|l|c|c|c|}
\cline { 2 - 4 } \multicolumn{1}{c|}{} & Pre-contrast & Gd-EOB-DTPA & $\boldsymbol{p}$-value \\
\hline Liver & $1020,099 \pm 69$ & $1138,681 \pm 81,3$ & $\mathrm{p}<0,001$ \\
\hline Pancreas & $1156,103 \pm 87,8$ & $1288,233 \pm 127$ & $\mathrm{p}=0,02$ \\
\hline Spleen & $778,723 \pm 44,7$ & $749,687 \pm 51,6$ & NS \\
\hline Renal cortex & $1746,148 \pm 66,5$ & $1791,255 \pm 58,1$ & NS \\
\hline Renal medulla & $1579,487 \pm 65,8$ & $1726,244 \pm 76$ & $\mathrm{p}<0,001$ \\
\hline Gallbladder & $2759,742 \pm 132,5$ & $2803,280 \pm 154,2$ & NS \\
\hline Muscle & $1339,112 \pm 72,5$ & $1377,131 \pm 78,9$ & NS \\
\hline Fat & $272,670 \pm 135,8$ & $348,428 \pm 147,6$ & NS \\
\hline
\end{tabular}
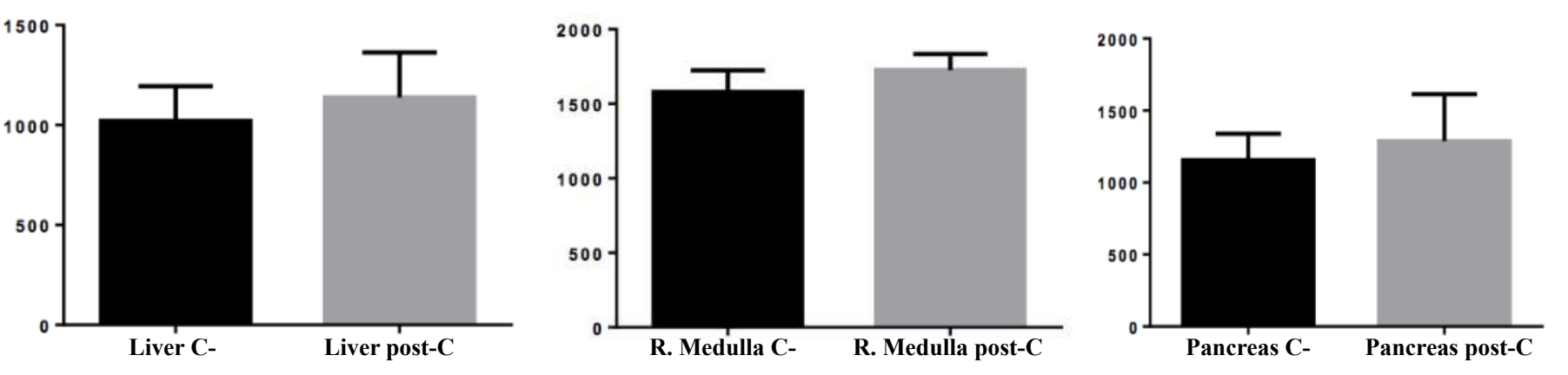

A plausible explanation of these quantitative changes should be related to the pharmacokinetics of Gd-EOB-DTPA, which alter ADC values of the organs which discharge these gadolinium chelates, such as liver and renal medulla. However, a statistically significant difference was observed also within the pancreatic parenchyma.

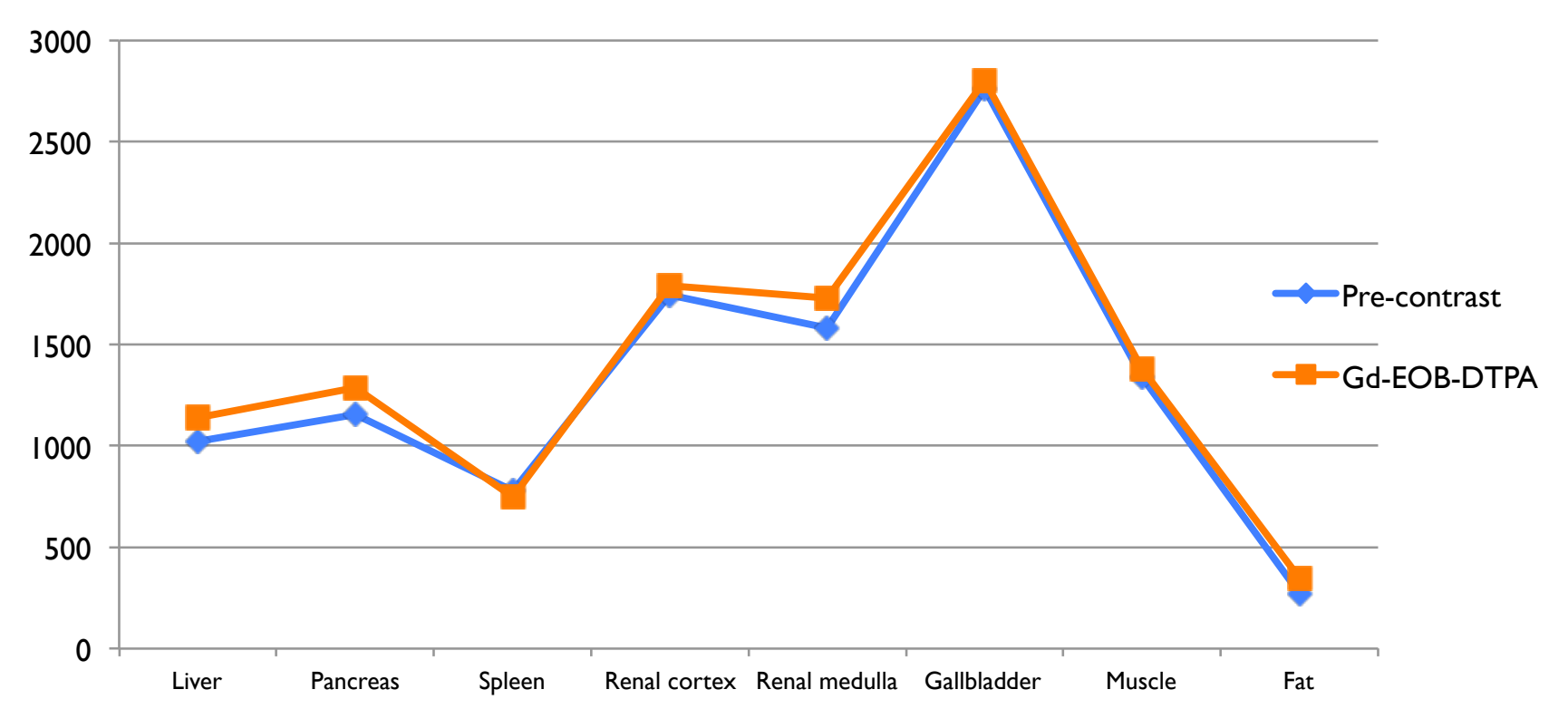

Concerning texture analysis, we did not observe any statistical difference in terms of mean values, variance, skewness and kurtosis before and after GdEOB-DTPA administration, except for liver skewness values $(\mathrm{p}=0,001)$.

\begin{tabular}{|l|c|c|c|c|c|c|c|c|}
\cline { 2 - 9 } \multicolumn{1}{c|}{} & \multicolumn{4}{c|}{ PRE-CONTRAST } & \multicolumn{4}{c|}{ GD-EOB-DTPA } \\
\cline { 2 - 9 } \multicolumn{1}{c|}{} & Mean & Variance & Skewness & Kurtosis & Mean & Variance & Skewness & Kurtosis \\
\hline Liver & 1285,87 & 13346,40 & $\mathbf{- 0 , 1 5 *}$ & $-0,13$ & 1252,20 & 11971,07 & $\mathbf{0 , 3 0} *$ & 0,24 \\
\hline Pancreas & 1426,08 & 20133,83 & 0,23 & $-0,33$ & 1439,56 & 25361,46 & 0,19 & $-47,28$ \\
\hline Spleen & 898,30 & 3940,92 & 0,11 & $-0,47$ & 863,29 & 4767,27 & 0,13 & $-0,49$ \\
\hline Renal cortex & 2009,75 & 23277,79 & $-0,41$ & 1,13 & 2122,71 & 21462,88 & $-0,13$ & 0,23 \\
\hline Renal medulla & 1895,58 & 13708,64 & 0,02 & $-0,17$ & 1980,20 & 10784,24 & 0,35 & 0,22 \\
\hline Gallbladder & 3146,83 & 26647,50 & 0,27 & 0,14 & 3054,10 & 8186,02 & 0,24 & $-0,31$ \\
\hline Muscle & 1501,39 & 12220,07 & $-0,03$ & $-0,44$ & 1544,77 & 13472,42 & $-0,09$ & $-0,02$ \\
\hline Fat & 521,66 & 27139,56 & 0,02 & $-0,02$ & 488,59 & 31162,88 & $-0,08$ & $-0,3$ \\
\hline
\end{tabular}

\section{Conclusion}

Diffusion weighted images and related ADC maps are not qualitatively influenced by Gd-EOB-DTPA. However, in our series Gd-EOB-DTPA significantly increases quantitative $\mathrm{ADC}$ values of liver, pancreas and renal medulla. These quantitative alterations should be considered when using numeric data calculated in the ADC maps.

Finally, Gd-EOB-DTPA does not modify the texture analysis of the normal tissues of splanchnic organs, except for an isolated increase of the liver skewness. 